\title{
Artritis séptica por Enterococcus faecalis en articulación nativa
}

\author{
Lucía Pomies ${ }^{1,2}$, Martín Elizondo ${ }^{1,2,3}$, Carolina Díaz-Cuñaa,2,4 \\ 'Servicio de Medicina Interna, Hospital Maciel. Facultad de Medicina. Universidad de la República, Montevideo, Uruguay \\ ${ }^{2}$ Servicio de Emergencia, Hospital Maciel. Montevideo, Uruguay \\ ${ }^{3}$ Servicio de Enfermedades hepáticas y Programa trasplante hepático, Hospital Militar, Montevideo, Uruguay \\ ${ }^{4}$ Servicio de Enfermedades autoinmunes, Hospital Maciel, Montevideo, Uruguay
}

Recibido: 19/06/2020

Aceptado: 02/09/2020

En línea: 31/12/2020

Citar como: Pomies L, Elizondo M, Díaz-Cuña C. Artritis séptica por Enterococcusfaecalis en articulación nativa. Rev Esp Casos Clin Med Intern (RECCMI). 2020 (Dic); 5 (3): 112-113. doi: 10.32818/reccmi.a5n3a4

Cite this as: Pomies L, Elizondo M, Díaz-Cuña C. Septic arthritis in native joint due to Enterococcus faecalis. Rev Esp Casos Clin Med Intern (RECCMI). 2020 (Dec); $5(3)$ : 112-113. doi: 10.32818/reccmi.a5n3a4

Autor para correspondencia: Carolina Díaz-Cuña. caroldiazcu@gmail.com

\section{Palabras clave \\ $\triangleright$ Artritis séptica \\ $\triangleright$ Articulación nativa \\ $\triangleright$ Enterococcus faecalis}

\section{Keywords}

$\triangleright$ Septic arthritis

$\triangleright$ Nativejoint

$\triangleright$ Enterococcus faecalis

\section{Resumen}

El Enterococcuss faecalis es un coco grampositivo, anaerobio facultativo. El reservorio más importante es el hombre dado que forma parte de la microbiota normal. Raramente es agente causal de artritis séptica debido a su baja afinidad por los tejidos osteoarticulares.

Presentamos un caso de un paciente de 81 años, con una uropatía obstructiva baja y sonda vesical que presenta artritis séptica sobre articulación nativa.

Abstract
Enterococcus faecalis is a gram-positive, anaerobic facultative coconut, the most important reservoir is the man because it
is part of the normal microbiota. It is rarely a causative agent of septic arthritis due to its low affinity for osteoarticular tissues.
We present an 81 -year-old patient, with a permanent bladder catheter for low obstructive uropathy, with diagnosis of
septic arthritis on a native joint.

Puntos destacados

$\triangleright$ El Enterococcus faecalis es un coco grampositivo que forma parte de la microbiota normal.

$\triangleright$ Es causa de infección urinaria, digestiva, bacteriemia, encontrándose raramente como agente causal de artritis séptica. Presenta baja afinidad por los tejidos osteoarticulares.

\section{Introducción}

El Enterococcus faecalis es un coco grampositivo, anaerobio facultativo, catalasa negativo, con capacidad de colonizar el tracto gastrointestinal. El reservorio más importante es el hombre dado que forma parte de la microbiota normal ${ }^{1,2}$. Es causa de infección urinaria, digestiva, bacteriemia y endocarditis.

Las etiologías más frecuentes de la artritis séptica (AS) en articulación nativa son Staphylococcus aureus, Streptoccocus spp seguida de bacilos gramnegativos. Los Enterococcus raramente son agentes causales de la AS, debido a su baja afinidad por los tejidos osteoarticulares ${ }^{1,2}$.

Reportamos un caso de AS aguda en articulación nativa por Enterococcus faecalis, cuya importancia radica en lo infrecuente de esta etiología.

\section{Caso clínico}

Antecedentes personales y enfermedad actual

Varón de 81 años con tabaquismo activo, diabetes mellitus dependiente de insulina, flutter auricular anticoagulado con warfarina. Uropatía obstructiva vinculada a hipertrofía prostática benigna con sonda vesical permanente, sin infecciones del tracto urinario previas, intervenido de meniscectomía de rodilla izquierda hacía 20 años.

Consulta por gonalgia izquierda, impotencia funcional y signos inflamatorios, sin fiebre. No ha tenido traumatismo o lesión previa.

\section{Exploración física}

En el examen físico se encontraba afebril, lúcido, eupneico y normotenso (Q SOFA=0); palidez cutáneo mucosa; ruidos cardíacos rítmicos de 76 cpm, y sin dolor, rubor, calor ni derrame articular en rodilla izquierda.

Pruebas complementarias

Los resultados de las pruebas complementarias fueron: hemoglobina 9,6 g/dL (13 g/dL) normocítica normocrómica, leucocitosis de 13.600/ $\mathrm{mm}^{3}\left(10.000 / \mathrm{mm}^{3}\right)$, 
proteína C reactiva $259 \mathrm{mg} / \mathrm{L}$ (3 mg/L), velocidad de sedimentación globular 100 $\mathrm{mm} / \mathrm{h}(20 \mathrm{~mm} / \mathrm{h})$, urea 1,91 mg/dL (0,4 mg/dL), creatinina 2,60 mg/dL (1,2 mg/ dL), INR 11 (1.3).

Urocultivo: flora polimicrobiana. Artrocentesis de rodilla izquierda diagnóstica y terapéutica: líquido de aspecto turbio, ligeramente hemático, amarillo lechoso.

Análisis del líquido articular: glucosa $<0,1 \mathrm{~g} / \mathrm{L}$ (0,3-0,6 g/L), proteínas totales $4,1 \mathrm{~g} / \mathrm{dL}$ (2,5 g/dL). 25.900 leucocitos $/ \mathrm{mm}^{3}$ (<200 leucocitos $/ \mathrm{mm}^{3}$ ), 88\% polimorfonucleares.

Se realizó radiografía que no mostró datos de osteomielitis.

Con diagnóstico de AS aguda, monoarticular, en articulación nativa, se realizó drenaje de cavidad articular y antibióticoterapia empírica con ciprofloxacina y clindamicina por vía endovenosa. Se aisló Enterococcus faecalis sensible a ampicilina y vancomicina tanto en hemocultivos como en el cultivo del líquido sinovial.

Un ecocardiograma transesofágico descartó compromiso endocárdico.

\section{Evolución}

Tras conocer la sensibilidad del microorganismo se desescaló antibiótico comenzando una estrategia antibiótica en monoterapia con betalactámicos, comenzando con ampicilina 2 g cada 4-6 h por vía endovenosa. La duración del tratamiento fue de 4 semanas, 21 días por vía parenteral y 10 días vía oral.

El paciente presentó una buena evolución clínica, con descenso de reactantes de fase aguda y sin secuelas funcionales a mediano plazo.

\section{Diagnóstico}

Artritis séptica por Streptococcus faecalis sobre articulación nativa.

\section{Discusión y conclusión}

Se diagnosticó monoartritis aguda, planteándose en primer lugar una artritis séptica, incluso sin presentar fiebre, ausente en más del 50\% de los casos $^{3}$. Los hemocultivos, el recuento leucocitario, los reactantes de fase aguda y la artrocentesis resultaron determinantes.

Si bien el diagnóstico de AS se realiza con un recuento de leucocitos en el líquido sinovial mayor que 50.000 células $/ \mathrm{mm}^{3}$, se han descrito casos de AS con un recuento leucocitario menor, siendo estos más frecuente en personas inmunocomprometidas ${ }^{4}$.

La existencia de hemocultivos positivos puede deberse a una infección hematógena con probable puerta de entrada en nuestro paciente en el tracto urinario, al ser portador de sonda vesical permanente, aunque no tiene antecedentes de infecciones urinarias previas.

Hay descritos pocos casos de AS por Enterococcus spp, siendo excepcional la causa por E. faecalis, y más aún en articulaciones nativas ${ }^{1}$. En una cohorte de pacientes con AS en articulación nativa, que valoró la epidemiología de la AS en 13 años de evolución, solo 3 de 71 pacientes afectados tuvieron infección por Enterococcus, sin especificarse la especie ${ }^{5}$

Otro estudio, realizado en un centro traumatológico de referencia en Francia, que incluyó 374 pacientes con AS durante un período de 30 años, identificó únicamente 6 casos de AS por Enterococcus ${ }^{6}$.
Se describen como factores de riesgo para presentar una AS por Enterococcus: presencia de múltiples comorbilidades, enfermedad renal crónica (ERC), insuficiencia cardíaca y diabetes mellitus. La articulación mayoritariamente afectada por este microorganismo es la rodilla $a^{5,6}$.

E. faecalis se presenta con mayor frecuencia en pacientes con prótesis osteoarticulares que sobre articulaciones nativas, dado la baja afinidad por los tejidos osteoarticulares de dicho microorganismo², predominando las infecciones polimicrobianas, siendo menos frecuentes las monomicrobianas como es el caso del paciente ${ }^{7}$. Presenta resistencia intrínseca a las cefalosporinas y es sensible a las penicilinas, glicopéptidos y aminoclucósidos². Por esta razón la ampicilina debería ser de primera elección frente a infecciones no endovasculares ni meníngeas por E. faecalis sensibles ${ }^{8}$

No disponemos de estudios controlados que nos orienten en la duración del tratamiento antibiótico que se debe realizar, siendo el período de tiempo en la mayoría de los trabajos de 4 semanas ${ }^{5}$.

En conclusión, si bien la AS es una patología frecuente en la práctica clínica habitual, la AS por E. faecalis es sumamente rara habiéndose descrito pocos casos en la literatura. Ante un paciente con AS pluripatológico con edad avanzada, diabetes mellitus, ERC y sonda vesical permanente, se debe sospechar otros microorganismos no habituales como los Enterococcus.

\section{Bibliografía}

1. Ramírez J, Toro M, Luque R. Artritis séptica por Enterococcus faecalis en paciente con catéter tunelizado para hemodiálisis. Reumatol Clínica 2007;3(4):193. https://linkinghub.elsevier.com/retrieve/pii/ S1699258X07736215.

2. Murray B. Tratamiento de infecciones por enterococos. Up to Date [Internet]. 2020;1-34. Available from: https://www.uptodate.com/contents/ treatment-of-enterococcal-infections?search=tratamiento de infecciones por enterococos\&source=search_result\&selectedTitle=1 150\&usag e_type=default\&display_rank=1.

3. García-Ramiro S, Monegal A, Bosch J, Soriano A. Protocolo de diagnóstico y tratamiento de la artritis séptica sobre una articulación nativa. Medicine 2014;11(59):3520-4. http://dx.doi.org/10.1016/S0304-5412(14)70809-1.

4. González Fernández JA, Noguera Pons JR, Tovar Beltrán JV, Navarro Blasco FJ. Artritis infecciosas. In: Castellano J, Roman J, Rosas J, editors. Enfermedades reumáticas: Actualización SVR, Valencia; 2008.p. 347-66.

5. Lotz H, Strahm C, Zdravkovic V, Jost B, Albrich W.C. Septic arthritis due to streptococci and enterococci in native joints: a 13 year retrospective study. Infection 2019; 47(5):761-770. https://doi.org/10.1007/s15010-01901301-w.

6. Dubost J, Couderc M , Tatar Z, Tournadre A, Lopez J, Mathieu S, et al. Threedecade trends in the distribution of organisms causing septic arthritis in native joints: Single-center study of 374 cases. Joint Bone Spine 2014; 81: 438-440. http://dx.doi.org/10.1016/j.jbspin.2014.05.001.

7. Tornero E, Senneville E, Euba G, Petersdorf S, Rodriguez-Pardo D, Lakatos $B$, et al. Characteristics of prosthetic joint infections due to Enterococcus sp. and predictors of failure: a multi-national study Clinical Microbiology and Infection 2014; 20 (11): 1219-1224. https://doi.org/10.1111/14690691.12721.

8. Bosch Mestres J, del Romero Guerrero J, Fresnadillo Martínez MJ, Garcia MI, García Sánchez E, Guerrero León MA, et al. Tratamiento de las infecciones producidas por microorganismos específicos. In: Mensa J, Gatell J M, Garcia Sanchez JE, Letang E, López Suñé E, Marco F, editors. Guía de terapéutica antimicrobiana, Barcelona; 2018. p.368-369. 